\title{
Oral rehabilitation of two siblings with Ectodermal Dysplasia : a case report
}

\author{
Dr. Amitava Bora ${ }^{1}$, Dr. Abhirup Goswami ${ }^{1}$, Dr. Sudipta Kar $^{2}$, \\ Dr. Gautam Kumar Kundu ${ }^{3}$ \\ ${ }^{1} 3^{\text {rd }}$ Year Postgraduate trainee, Department of Pedodontics and Preventive Dentistry, Guru Nanak Institute of \\ Dental Sciences and Research, Kolkata, India \\ ${ }^{2}$ Senior Lecturer, Department of Pedodontics and Preventive Dentistry, Guru Nanak Institute of Dental \\ Sciences and Research, Kolkata, India \\ ${ }^{3}$ Professor and Head of the Department, Department of Pedodontics and Preventive Dentistry, Guru Nanak \\ Institute of Dental Sciences and Research, Kolkata, India
}

\begin{abstract}
Ectodermal dysplasia (ED) is a congenital syndrome characterised mainly by abnormalities of two or more tissues of ectodermal origin namely skin, nails, hair and teeth. Two brothers of 14 years and 10 years of age respectively reported with chief complaint of multiple missing teeth. Thorough medical and dental examinations were performed and findings were in accordance with the typical features of Ectodermal Dysplasia. Pedigree analysis, dermatoglyphic analysis and skin biopsy were also performed. The elder brother was treated with a maxillary removable overdenture and a mandibular removable partial denture. In the younger brother aesthetic composite restoration was done in maxillary anteriors followed by placement of maxillary and mandibular removable partial dentures.
\end{abstract}

Key words: Ectodermal Dysplasia, Oligodontia, Prosthesis.

\section{Introduction}

Ectodermal dysplasia (ED) is a congenital syndrome characterised mainly by tissue abnormalities of ectodermal origin namely skin, nails, hair and teeth. ${ }^{1}$ To date, more than 192 distinct types have been described in literature, among them most common are X-linked recessive hypohidrotic ectodermal dysplasia (ChristSiemens-Touraine syndrome), and hidrotic ectodermal dysplasia (Clouston syndrome). ${ }^{2}$ The prevalence of hypohidrotic ectodermal dysplasia, the most common variant, is estimated to be 1 per 100,000 births. $^{3}$ The intraoral menifestations of this syndrome include anodontia or hypodontia of the primary and/or permanent teeth, hypoplastic conical teeth, and underdevelopment of the alveolar ridges. ${ }^{4}$ Factors such as patient's chronological, skeletal and dental maturity in conjunction with no of missing teeth, soft tissue defects, existence of malformed dentition and psychological status must be considered in the treatment planning. ${ }^{5}$ Timely diagnosis is very important as this facilitates early dental, physical, social as well as psychological rehabilitation. This article aims to present clinical features, diagnostic methods and dental management of ectodermal dysplasia through a case report.

\section{Case Report}

Two brothers of 14 and 10 years of age (Fig.1 \&2) respectively reported to the Out Patient Department of Pedodontics and Preventive Dentistry of Guru Nanak Institute of Dental Sciences and Research, Kolkata, India with the chief complaint of multiple missing teeth, masticatory difficulty and unaesthetic appearance. Medical and Dental history was taken and a thorough clinical examination was performed.

History revealed both brothers were born by normal delivery. There was no significant family history present. Both brothers gave history of lack of sweating, dryness of skin, raised body temperature and recurrent cough and cold.

Extraoral examination (Fig. 1 \&2) revealed protuberant supraorbital ridge, depressed midface, dry and everted lips, shunken cheeks, enlarged ears, wrinkling on the forehead, prominent chin, and pointed nose tip for both the brothers. Both had atrophied nails, thick and hyperkeratotic skin (Fig. $3 \& 4$ ), adequate but very thin hair on head which appeared fine in texture.

Intraoral examination revealed, the elder sibling had dentition of 11,53,54,55,21,53,55,34,65,36,44,75. 11 and 21 were conical in shape and hypoplastic in nature. The dentition for younger sibling was $11,53,54,55,16,21,63,64,65,26,71,34,75,36,81,82,83,44,85,46$. Large midline diastema was present between 11 and $21.71,81$ and 82 were hypoplastic and decayed. Underdeveloped alveolar ridge and clinically normal salivary secretion was found in both the brothers. Orthopantamogram (Fig.5) revealed poor bony support for both and impacted 43 in case of elder sibling. 
Both the patients were referred to Department of Medicine of Guru Nanak Institute of Dental Sciences and Research, Kolkata for detailed systemic examination. Respiratory examination revealed the younger sibling had recurrent upper respiratory tract infection and allergic rhinitis. Cardiovascular, Gastrointestinal, Ophthalmological and Otolaryngological examinations did not reveal any pathological finding for both the brothers.

A skin punch biopsy was performed from hypothenar eminence of right palm of elder brother that revealed hyperkeratosis and mild acanthosis in epidermis and absence of sebaceous glands in dermis. The hair follicles and the sweat glands were rudimentary (Fig.6). As the younger brother was not very cooperative it was not possible to perform skin biopsy for him.

Pedigree analysis was done and pedigrees were drawn up to three generations, but no positive correlation was found. Dermatoglyphic studies were carried out using hand imprints. The hand impressions (Fig.7) were taken on white sheet using stamp ink pad. Hand impressions of two unaffected children of same age group were also taken as control. The imprints were evaluated using hand magnifying lens. The affected brothers showed a number of variations like presence of flattened and vestigial palmer ridge patterns. The 'atd' angle (atd angle is an angle formed by the tri axial radius and the tri radii at the base of the index finger and little finger) ${ }^{8}$ was found to be higher in study group than control group.

Treatment plan was made based on the clinical, histological and radiological examinations. Hand wrist radiographs of both the subjects were taken and after studying the skeletal maturation indicators as given by Fishman, it was decided that no fixed prosthesis could be planned for both the brothers as the skeletal maturation was incomplete. The elder brother was given a maxillary transitional overdenture taking support from 11 and 21 and a mandibular removable partial denture (Fig.8). 65 and 75 were restored with stainless steel crown as they were hypoplastic. In younger brother maxillary anterior diastema was closed by direct light cure composite restoration. 71, 81 and 82 were also restored with light cure composite. Removable partial dentures were fabricated for both maxillary and mandibular arch in order to improve mastication and aesthetics (Fig.8). Post operative instructions and oral hygiene instructions were given. Recalls were scheduled every 6 months to evaluate jaw development and teeth eruption.

\section{Discussions}

Thurnam ${ }^{6}$ published the first clinical case report of ectodermal dysplasia in 1848. The term was first coined in 1929 by Weech. ${ }^{7}$ Pure ectodermal dysplasias are manifested by defects in ectodermal structures alone, while ectodermal dysplasia syndromes are defined by the combination of ectodermal defects in association with other anomalies. $^{7}$

Two types of inheritance for this disorder are postulated. An X-linked recessive inheritence where only males are affected and an autosomal dominant inheritance where both males and females are affected. ${ }^{7}$

Most important features may include the following:

- Dry, hypopigmented, eczematous skin.

- Sweating, lacrimation and salivation may be absent or reduced.

- Sparse, thin and brittle hair with alopecia and sparse or absent eyebrows, eyelashes and body hair.

- Nail dystrophy.

- Dental features may include hypodontia or anodontia; malformed, rudimentary, or pegged teeth; and/or enamel defects and frequent dental caries.

- Impaired respiratory, gastrointestinal and immune system functioning.

Laboratory investigations include radiological examinations like orthopantamogram, $\mathrm{x}$ rays of hand and feet, CT scan. Sweat pore counts, pilocarpine iontophoresis, and skin biopsy may document hypohidrosis and a reduction in the number of eccrine glands. ${ }^{7}$ Dermatoglyphic studies show disruption of dermal ridges due to decrease in number of sweat pores. ${ }^{8}$ Slight variation in 'atd' angle was noted when compared to normal individuals, increase in number of arches and decrease in number of radials were also noted. Prenatal diagnosis using genetic mutation analysis and karyotyping may be performed for those patients with ectodermal dysplasia whose genetic mutation is known.

There is no definite time to begin treatment but according to Till and Marques ${ }^{9}$ initial prosthesis should be delivered when the child starts going to school. Treatment of ectodermal dysplasia patients involves a multidisciplinary approach. Oral and dental rehabilitation of patients with ectodermal dysplasia is necessary to improve skeletal relationships during craniofacial growth and development as well as to improve aesthetics, speech, and masticatory efficiency. ${ }^{2}$ Overdentures, removable complete dentures and partial dentures are often part of treatment for patients with ectodermal dysplasia. ${ }^{10}$ In recent years endosseous implants have been recognized as an important alternative for ectodermal dysplasia patients to support, stabilize and retain the prosthesis. ${ }^{11}$ Considering the age and potential growth of patients fixed treatment option with endoosseous implant was postponed. 
In the present case, a transitional removable overdenture and removable partial dentures were placed which will not impair skeletal growth. Osseo integrated implants and permanent prosthodontic treatments are planned after completion of skeletal maturity. Periodic recall for young patients with ectodermal dysplasia is very important because prosthetic modification and replacement may be needed as a result of continuing growth and development.

\section{Conclusion:}

Management of clinical manifestations associated with ectodermal dysplasia presents a unique challenge for pedodontists. Treatment of young partially edentulous patients with removable partial or over denture is an acceptable, available and cost effective modality, which improves function, speech, aesthetics and Psychosocial condition. However, its long term success depends on regular recall appointments and meticulous maintenance of oral and prosthetic hygiene.

\section{References}

[1]. Suri S, Carmichael RP and Tompson BD. Simultaneous functional and fixed appliance therapy for growth modification and dental alignment prior to prosthetic habilitation in hypohidrotic ectodermal dysplasia: a clinical report. J. Prosthet Dent. 2004; 92: 428433 .

[2]. Tarjan I, Gabris K, and Rozsa N. Early prosthetic treatment of patients with ectodermal dysplasia: a clinical report. Journal of Prosthetic Dentistry, vol. 93, no. 5, pp. 419-424, 2005.

[3]. Wisniewski SA, Kobielak A, Trzeciak WH, Kobielak K. Recent advances in understanding of the molecular basis of anhidrotic ectodermal dysplasia: discovery of a ligand, ectodysplasin A and its two receptors. Journal of Applied Genetics, vol. 43, no. 1, pp. 97-107, 2002.

[4]. Kearns G, Sharma A, Perrott D, Schmidt B, Kaban L, Vargervik K. Placement of endosseous implants in children and adolescents with hereditary ectodermal dysplasia. Oral Surg Oral Med Oral Pathol Oral Radiol Endod 1999; 88:5-10.

[5]. Hobkirk JA, Nohl F, Bergendal B, Storhaug K, Richter MK. The management of ectodermal dysplasia and severe hypodontia. International conference statements. Journal of Oral Rehabilitation, vol. 33, no. 9, pp. 634-637, 2006.

[6]. Tarjan I, Gabris K, Rozsa N. Early prosthetic treatment of patients with ectodermal dysplasia: a clinical report. J. Prosthet Dent. 2005; 93: 419-424.

[7]. Shah KN. Ectodermal Dysplasia [Internet]. [Updated 2014 Feb 14; cited 2014 March]. Available from: http://emedicine.medscape.com/article/1110595-overview.

[8]. Sidhu M, Kale DA, Kotrashetti SV. Karyotyping, dermatoglyphic, and sweat pore analysis of five families affected with ectodermal dysplasia. J Oral Maxillofac Pathol. 2012 Sep-Dec; 16(3): 380-387.

[9]. Till MJ, Marques AP. Ectodermal Dysplasia: Treatment considerations and case reports. Northwest Dent 1992; 71 : 25-28.

[10]. Pigno MA, Blackman RB, Cronin RJ, Cavazos E. Prosthodontic management of ectodermal dysplasia: a review of the literature. J. Prosthet. Dent. 1996; 76: 541-545.

[11]. Imirzalioglu P, Uckan S, Haydar SG. Surgical and prostho-dontic treatment alternatives for children and adolescents with ectodermal dysplasia: a clinical report. J Prosthet Dent 2002;88:569-572

\section{Figures}

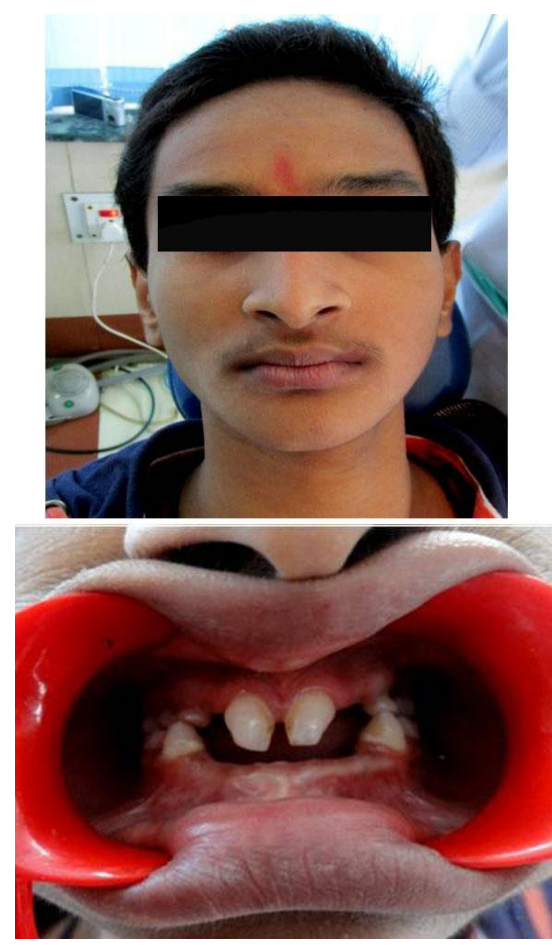

Figure 1: Extra oral and Intra oral view of elder brother 


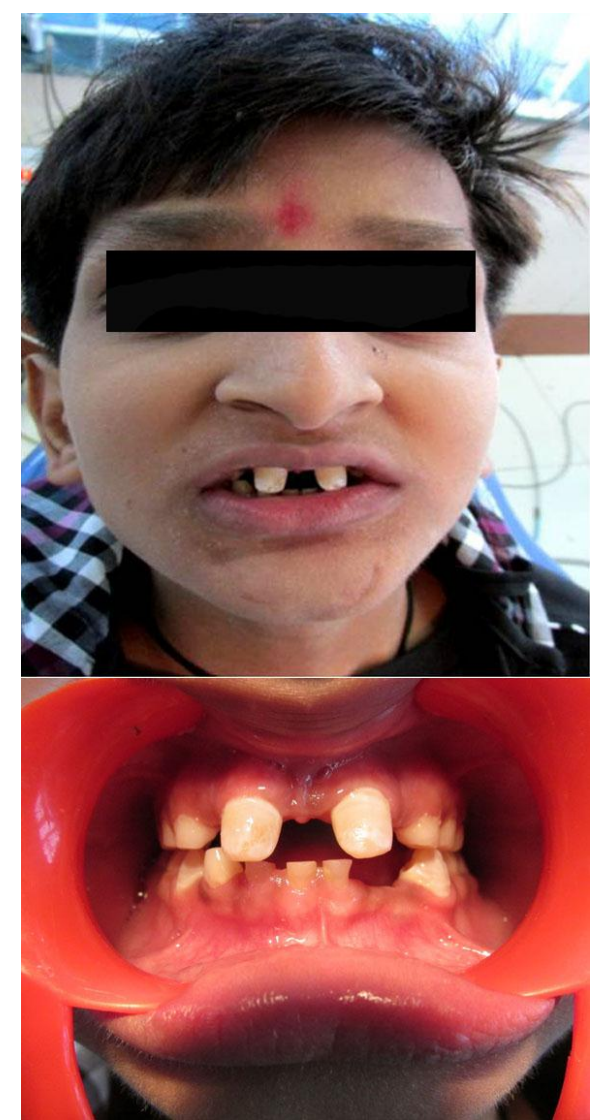

Figure 2: Extra oral and Intra oral view of younger brother
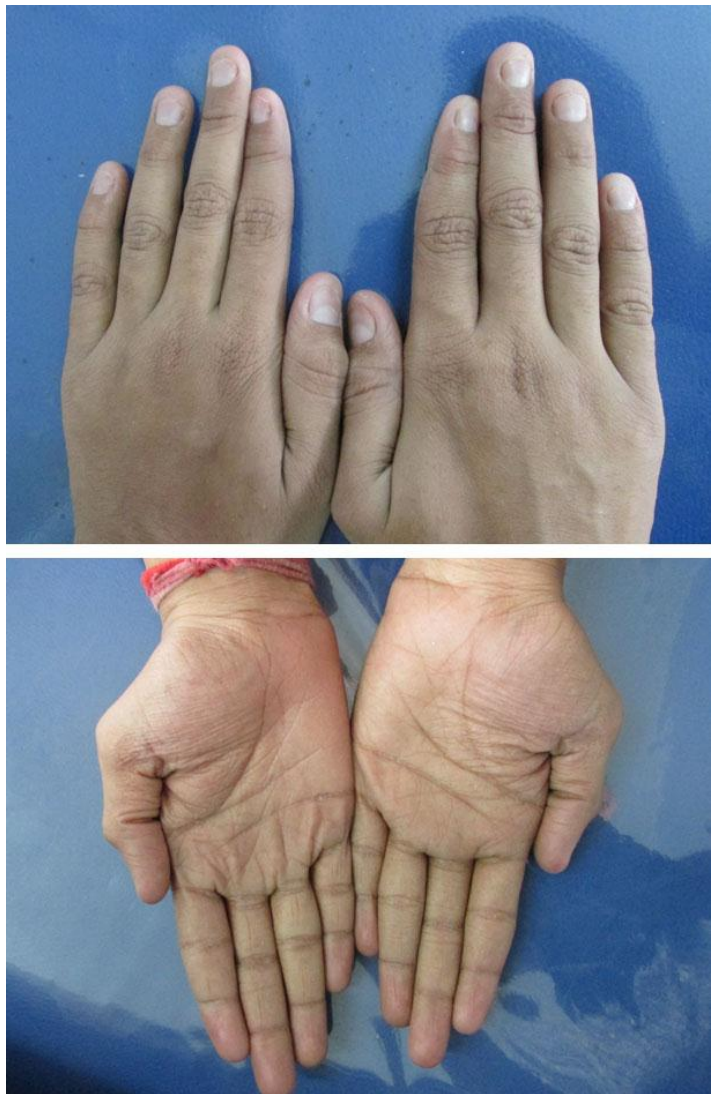

Figure 3: Atrophic nails and hyperkeratotic skin of elder brother 


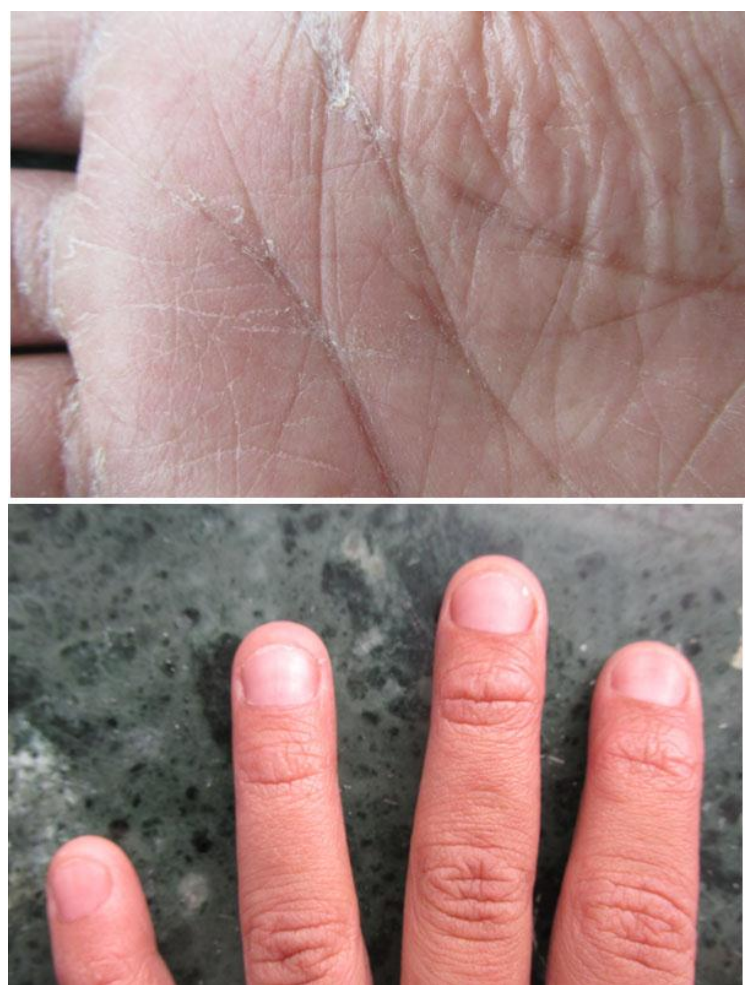

Figure 4: Atrophic nails and hyperkeratotic skin of younger brother
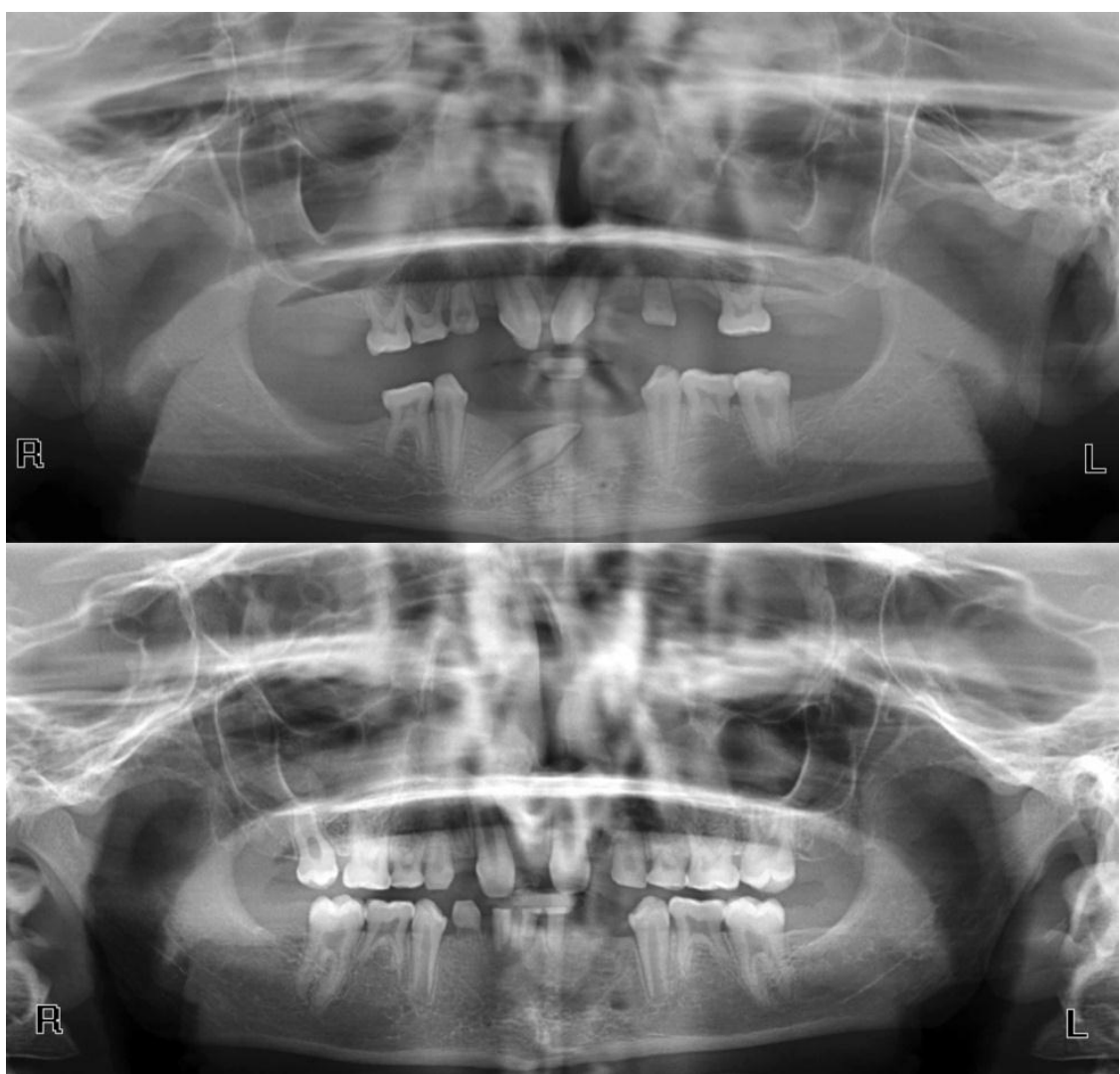

Figure 5: Panoramic radiographs of elder brother (above) and younger brother (below) 


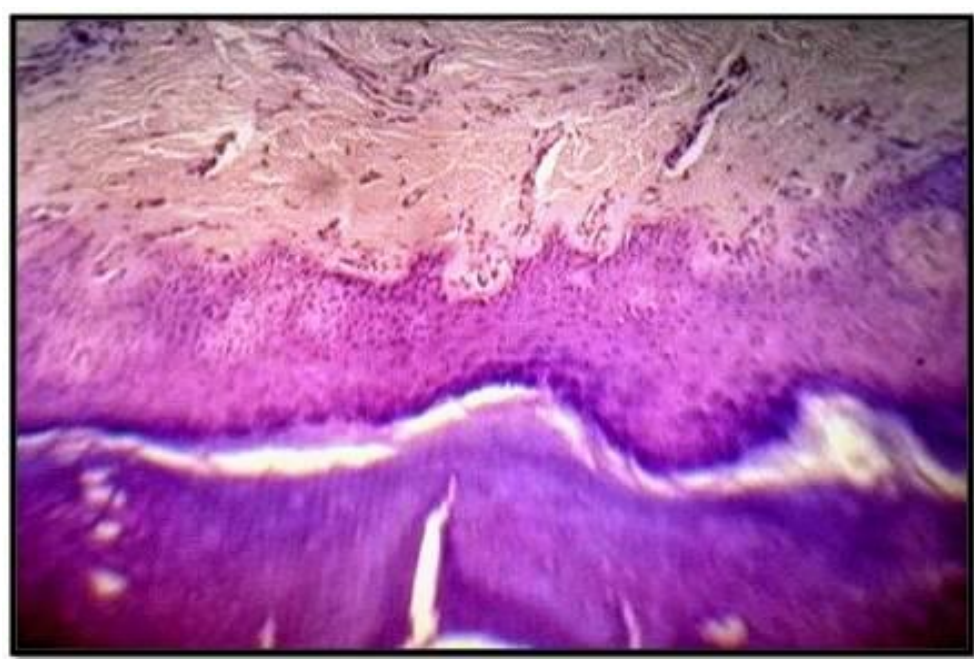

Figure 6: Photomicrograph of histological section from skin of hypothenar eminence of elder brother

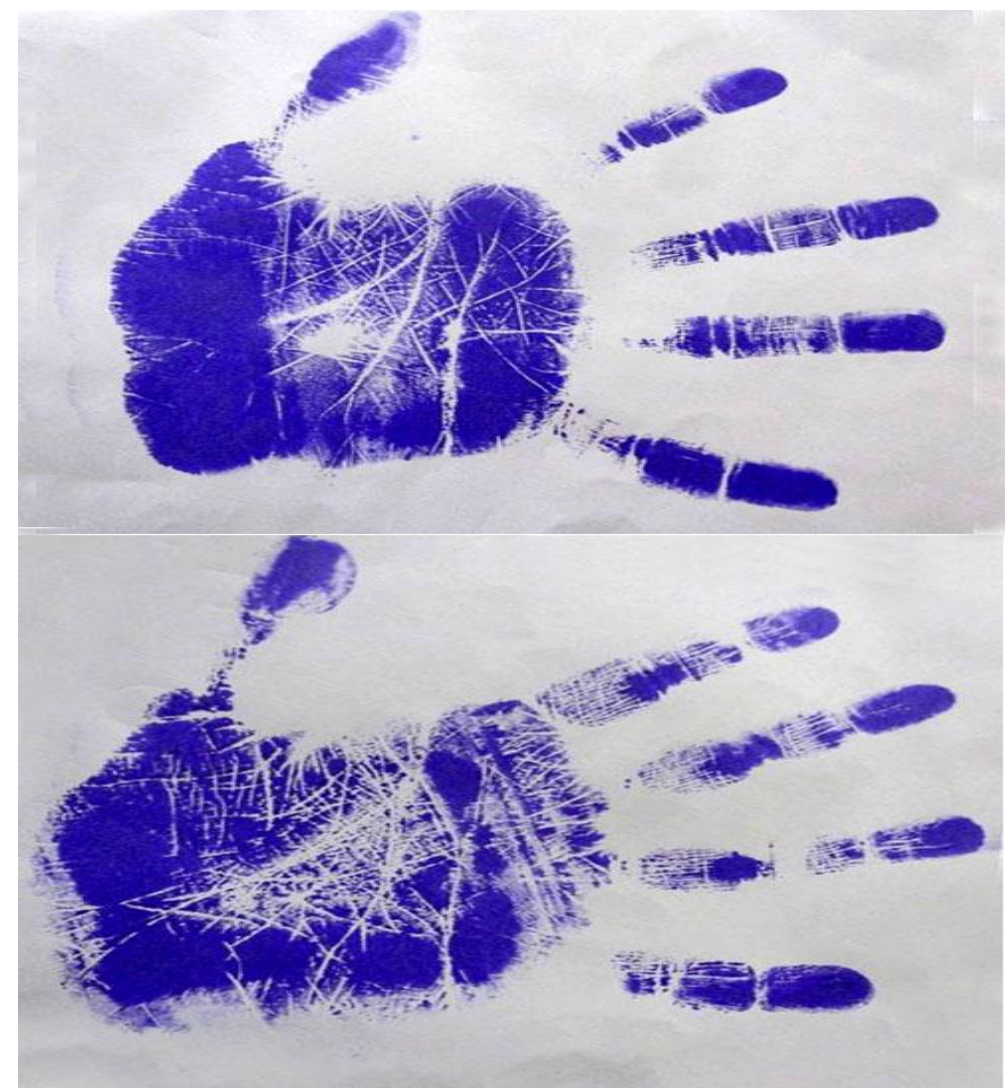

Figure 7: Hand impressions of elder brother (above) and younger brother (below) showing flattened palmer ridge pattern and higher 'atd' angle 


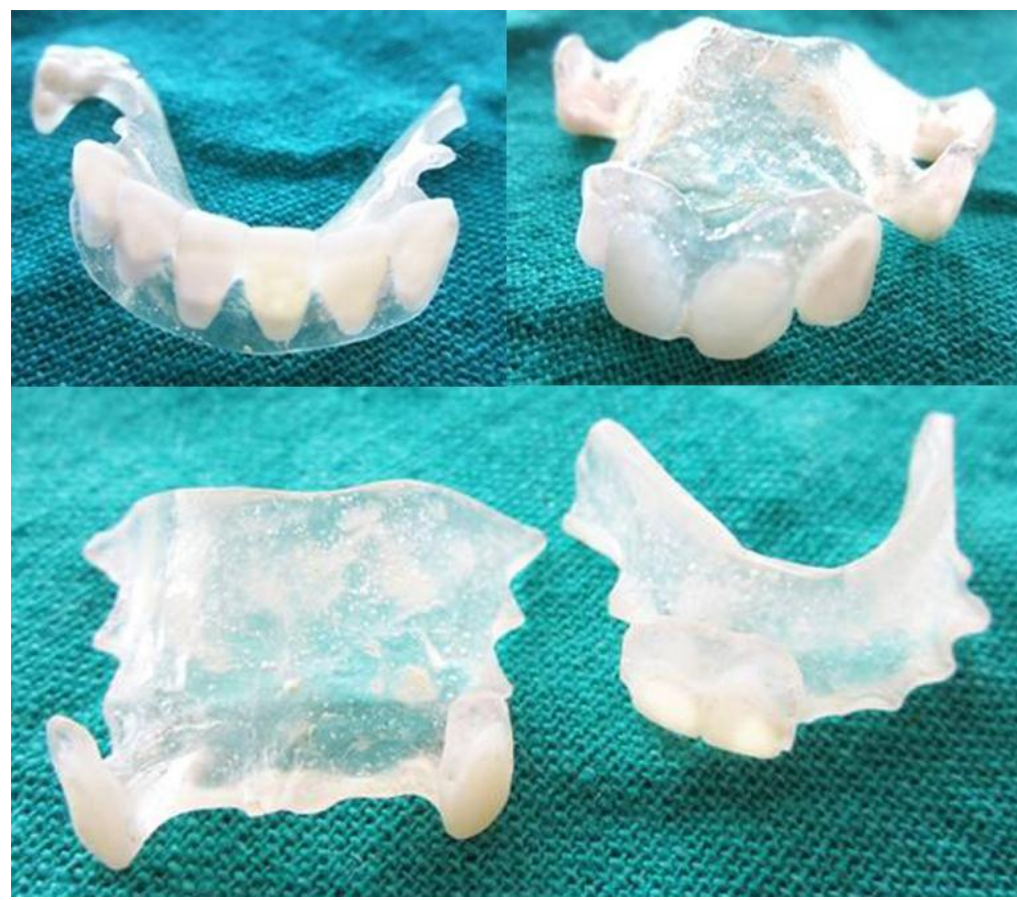

Figure 8: Removable prosthetic appliances for elder brother (above) and younger brother (below)
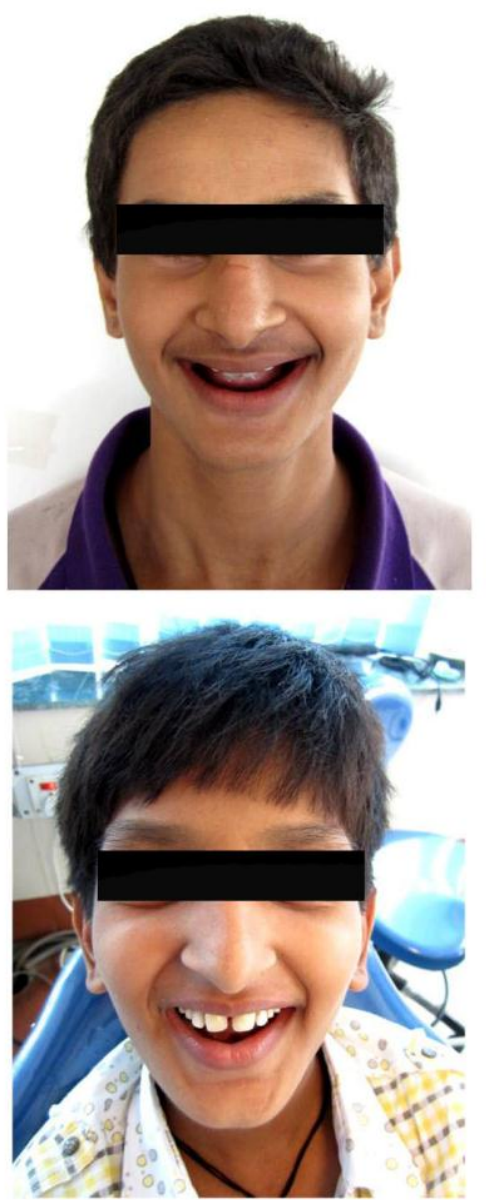

Figure 9: Post operative view of elder brother (above) and younger brother (below) 